\title{
A systematic comparison of treatment modalities for nasal extranodal natural killer/T-cell lymphoma in early stages between concurrent chemoradiotherapy and sequential chemotherapy
}

\author{
This article was published in the following Dove Press journal: \\ OncoTargets and Therapy \\ 20 July 2017 \\ Number of times this article has been viewed
}

\author{
Ming Qian' \\ Hengmin Tao' \\ Wei $\mathrm{Xu}^{2}$ \\ Hongzhi ji² \\ 'Department of Head and Neck \\ Radiotherapy, Shandong Provincial \\ Hospital Affiliated to Shandong \\ University, Jinan, Shandong, People's \\ Republic of China, ${ }^{2}$ Department \\ of Otorhinolaryngology-Head and \\ Neck Surgery, Shandong Provincial \\ Hospital Affiliated to Shandong \\ University, Jinan, Shandong, People's \\ Republic of China
}

Aim: Nasal extranodal natural killer/T-cell lymphoma (nasal ENKTL), which is strongly associated with the Epstein-Barr virus infection, is a common disease in Asia and Latin America. We conducted a retrospective study to compare the overall survival (OS) following concurrent or sequential treatment with radiotherapy and chemotherapy in patients with early stage ENKTL.

Patients and methods: The records of 58 cases from between 2000 to 2010 were retrieved. Of these, 28 patients (15 males, with median age of 51 years) were treated with sequential chemotherapy followed by radiotherapy (SCRT) and 30 patients (17 males, with median age of 46 years) were treated with concurrent chemoradiotherapy (CCRT). Subsequently, the OS, 5-year progression-free survival (PFS), 5-year locoregional-free survival (LRFS), and relevant toxicities were analyzed.

Results: There were no significant differences in the toxicities and complete response rate between the 2 groups (all $P>0.05$ ) during and immediately after treatment. Kaplan-Meier curve analysis demonstrated that there were significant differences between the CCRT and SCRT groups with regard to 5 -year OS $(72.9 \%$ vs $47.1 \%, P=0.029)$, 5-year PFS $(68.8 \%$ vs $34.2 \%$, $P=0.030)$, and LRFS (78.9\% vs $45.7 \%, P=0.026$ ).

Conclusion: We have demonstrated that in comparison with SCRT, CCRT significantly improves the survival outcome in patients with localized ENKTL, with acceptable toxicities. Further clinical trials are needed.

Keywords: nasal extranodal natural killer/T-cell lymphoma, concurrent chemoradiotherapy, treatment outcome

\section{Introduction}

Extranodal natural killer (NK)/T-cell lymphoma (ENKTL), nasal type, is a highly aggressive and heterogeneous disease, more prevalent in Asia than in Western countries. It has been reported that its frequency among all malignant lymphomas is $\sim 3 \%-10 \%$ in East Asia but $<1 \%$ in Western countries. ${ }^{1,2}$ ENKTL is the most common peripheral T-cell lymphoma in China, with an incidence as high as $11 \%$ of all lymphoma. ${ }^{3,4}$ Even though two-thirds of ENKTL patients present with localized disease, prognosis remains dismal. ${ }^{5}$ ENKTL is characterized by the expression of Epstein-Barr virus (EBV) and NK/T-cell markers (CD56/CD3 and cytotoxic molecules). Circulating cell-free EBV-DNA levels can be detected using real-time quantitative reverse transcription-polymerase chain reaction (RT-PCR) in the blood
Correspondence: Ming Qian

Department of Head and Neck Radiotherapy, Shandong Provincial Hospital Affiliated to Shandong University, 324 Jingwuweiqi Road, Huaiyin, Jinan, Sandong 25002I, People's Republic of China

Tel +86 53I 6877 85I2

Email shenglixiyuanqianm@।63.com 
of patients with ENKTL. Previous studies have demonstrated that the EBV-DNA load correlates with the clinical stage and can be used to monitor disease progression and to predict prognosis in patients. ${ }^{6,7}$

As ENKTL is relatively sensitive to radiation, radiotherapy (RT) alone is an effective treatment. First-line RT has been considered the initial treatment of choice for localized ENKTL. ${ }^{8,9}$ Regrettably, RT alone is insufficient to improve survival due to a high incidence of disease progression. Chemotherapy alone results in poor outcomes and may be inferior to RT alone. ${ }^{10,11}$ However, it is vital even for early-stage ENKTL. Two prospective trials, conducted in Japan ${ }^{12}$ and Korea, ${ }^{13}$ have shown promising outcomes, with an overall survival (OS) of 70\%-80\% using concurrent chemoradiotherapy (CCRT) for localized ENKTL. Based on these results, CCRT has become the standard treatment of localized ENKTL. ${ }^{12,13}$ It is well accepted that combining the 2 therapies can improve treatment efficacy. To date, however, no standard therapeutic approach has been established for this aggressive disease, due to the lack of data from prospective studies. Therefore, the appropriate mode of combined modality therapy for chemotherapy and RT is not clearly defined. In the present study, we report our experience comparing the efficacy of sequential chemotherapy followed by radiotherapy (SCRT) and CCRT in a cohort of 58 patients with ENKTL.

\section{Patients and methods Patients}

The present retrospective cohort is composed of 58 newly diagnosed and previously untreated patients with histopathologically confirmed ENKTL, stage I/II. The patients received radiochemotherapy in Shandong Provincial Western Hospital between January 2004 and December 2010. The 58 patients were treated with the same chemotherapy regimen of L-CHOP (L-asparaginase $6,000 \mu / \mathrm{m}^{2}$ days $2-8$, cyclophosphamide, $750 \mathrm{mg} / \mathrm{m}^{2}$ day 1 , vincristine, $1.4 \mathrm{mg} / \mathrm{m}^{2}$ day 1 [maximal dose $2 \mathrm{mg}$ ], doxorubicin $50 \mathrm{mg} / \mathrm{m}^{2}$ day 1 , and dexamethasone $10 \mathrm{mg}$ days $1-8$ ). Of these, 30 patients were treated with CCRT and 28 with SCRT. Approval for this study was obtained from the ethical committee of Shandong Provincial Western Hospital. Written informed consent was obtained from all participants.

The inclusion criteria of this retrospective study were as follows:

1. Pathologically confirmed diagnosis of ENKTL based on the WHO classification of Tumours of Haematopoietic and Lymphoid Tissues.
2. Histopathological examination showing the features of angiocentric infiltration, necrosis, and polymorphism of individual cells.

3. Immunohistochemical studies were performed on the following markers: CD45, CD43, CD45RO, CD2, CD3, CD79, CD20, TIA, and other markers. All patients were positive for at least $1 \mathrm{NK}$ or T-cell marker and negative for B-cell markers. Bone marrow Epstein-Barr virusencoded small RNA (EBER) staining and EBV DNA blood levels were not routinely performed in every case.

\section{Treatment}

A 3-field 3-dimensional conformal radiotherapy (3D-CRT) technique using 1 anterior portal and 2 lateral fields with $6 \mathrm{MV}$ was applied. In addition, an appropriate 9 or $12 \mathrm{MeV}$ electron beam was used to compensate for the lower dose to the anterior ethmoid. For intensity-modulated radiotherapy (IMRT) planning, 5-7 fields were given. Radiation for primary tumors was given in 2.0 Gy fractions every day, 5 days a week, for 4-6 weeks, for a total dose of 40-60 Gy. Definitive RT was conducted using 3D-CRT and IMRT. The gross tumor volume (GTV) was determined according to computed tomography (CT) or magnetic resonance imaging (MRI) analysis and endoscopic findings. The clinical target volume (CTV) consisted of the GTV and adjacent structures with potential involvement. If the neck lymph nodes were involved, the ipsilateral neck was irradiated. No prophylactic cervical irradiation was delivered. The CTV was expanded by $5 \mathrm{~mm}$ to obtain the planning target volume (PTV). In the CCRT group, 20 cases were treated with IMRT and 10 cases were treated with 3D-CRT. In the SCRT group, 18 cases were treated with $3 \mathrm{D}-\mathrm{CRT}$ and 10 cases were treated with IMRT.

In the SCRT group, 2-6 cycles of L-CHOP regimen were given as front-line treatment, followed by RT. The number of CHOP cycles depends on the chemotherapy efficacy. If a complete response $(\mathrm{CR})$ or partial response $(\mathrm{PR})$ can be achieved, 4-6 cycles of CHOP will be administered before radiation therapy. If a PR cannot be reached after 2-4 cycles of CHOP, the patients will be transferred for RT.

In the CCRT group, CCRT was initiated on the first day of RT. During the period of RT, 2 cycles of L-CHOP were delivered, followed by 2-4 cycles of CHOP after RT.

\section{Evaluation of the treatment response}

The treatment response was assessed by clinical symptoms, sign, and radiographic image according to the international revised response criteria of lymphoma. CR was defined as 
disappearance of all evidence of disease. PR was defined as a $50 \%$ decrease in measurable disease. Stable disease (SD) was defined as a decrease of $<50 \%$ or an increase of $<25 \%$ in tumor size, and progressive disease (PD) was defined as a $>25 \%$ increase in tumor size. Local recurrences and distant metastases were documented by biopsy or imaging studies.

\section{Follow-up and statistical analyses}

After completion of treatment, patients were followed up as outpatients by their oncologist. Each follow-up visit consisted of a physical examination, complete blood count, serum biochemistry including lactate dehydrogenase (LDH) levels, and $\mathrm{CT}$ or MRI of the head and neck region. Follow-up visits were conducted every 3 months for the first 2 years, every 6 months for the next 3 years, and annually after the initial 5 years. The primary end point included objective response, OS, progression-free survival (PFS), and locoregional-free survival (LRFS). The OS was observed from the first day of treatment until death or the most recent follow-up time. The PFS was observed from the first day of treatment until progression, death, or the most recent follow-up time, and LRFS was observed from the first day of treatment until recurrence, death, or the most recent follow-up time. All statistical analyses were performed using SPSS 17.0 software. The chi-square test was used to calculate statistical group comparisons of categorical variables. Survival analysis was performed using the Kaplan-Meier method, and comparisons were calculated using the log-rank test. $P<0.05$ was considered statistically significant, and all $P$-values correspond to 2-sided significance tests.

\section{Results}

\section{Patient characteristics}

The baseline characteristics of all patients are shown in Table 1, and there were no significant differences of these characteristics between the 2 groups (Table 1).

\section{Short-term outcome}

In the 30 patients in the CCRT group, CR, PR, and PD were observed in $24(80.0 \%)$ patients, $5(16.7 \%)$ patients, and 1 (3.3\%) patient, respectively, after the completion of CCRT. Of the 28 patients who received SCRT, 6 (21.4\%) achieved a CR and 10 (35.7\%) patients achieved PR, whereas the remaining 12 patients (42.9\%) showed SD or PD after initial chemotherapy. Of the 22 patients with PR, SD, and PD, 19 had persistent disease, regrowth, or progression of local disease and 3 developed systemic progression. However, 14 of these 22 patients with PD after L-CHOP achieved CR
Table I Clinical characteristics of patients with ENKTL, nasal type

\begin{tabular}{|c|c|c|c|c|}
\hline Characteristics & $\begin{array}{l}\text { CCRT } \\
(n=30)\end{array}$ & $\begin{array}{l}\text { SCRT } \\
(n=28)\end{array}$ & $\chi^{2}$ value & $P$-value \\
\hline Sex & & & 0.056 & 0.813 \\
\hline Male & 17 & 15 & & \\
\hline Female & 13 & 13 & & \\
\hline Age (years) & & & 0.554 & 0.457 \\
\hline$\leq 60$ & 21 & 17 & & \\
\hline$>60$ & 9 & 11 & & \\
\hline \multicolumn{2}{|c|}{ ECOG performance status } & & 1.353 & 0.336 \\
\hline $0-1$ & 22 & 24 & & \\
\hline$\geq 2$ & 8 & 4 & & \\
\hline Ann Arbor stage & & & 0.322 & $0.57 \mid$ \\
\hline IE & 16 & 17 & & \\
\hline IIE & 14 & 11 & & \\
\hline B symptoms & & & 0.030 & 0.826 \\
\hline Yes & 7 & 6 & & \\
\hline No & 23 & 22 & & \\
\hline LDH level & & & 1.283 & 0.325 \\
\hline Normal & 26 & 21 & & \\
\hline Increased & 4 & 7 & & \\
\hline Primary site & & & 0.588 & 0.745 \\
\hline Left nasal cavity & 12 & 14 & & \\
\hline Right nasal cavity & 14 & 11 & & \\
\hline Bilateral nasal cavity & 4 & 3 & & \\
\hline IPI score & & & 0.433 & 0.805 \\
\hline 0 & 17 & 15 & & \\
\hline I & $\mathrm{II}$ & 12 & & \\
\hline $2-3$ & 2 & 1 & & \\
\hline
\end{tabular}

Abbreviations: ENKTL, extranodal natural killer/T-cell lymphoma; CCRT, concurrent chemoradiotherapy; SCRT, sequential chemotherapy followed by radiotherapy; ECOG, Eastern Cooperative Oncology Group; LDH, lactate dehydrogenase; IPI, International Prognostic Index.

after completion of subsequent RT. There was no significant difference in the $\mathrm{CR}$ rates between the 2 groups (CCRT $80.0 \%$ vs SCRT $71.4 \%, P=0.446$ ).

\section{Survival outcome}

The median follow-up period was 70.6 months (range, 12-100 months) for all patients, and the median follow-up period for patients receiving CCRT was 79.8 months (range, 24-100 months) and for patients receiving SCRT was 57.8 months (range, 12-87 months). The 5-year OS rate was significantly better in CCRT group than in SCRT group ( $72.9 \%$ vs $47.1 \%, P=0.045)$, and the 5 -year PFS was also better in CCRT group than in SCRT group (64.2\% vs $34.2 \%, P=0.046$ ) (Figures 1 and 2 ).

A total of 25 patients ( 10 of 30 [33.3\%] in the CCRT group and 15 of 28 [53.6\%] in the SCRT group) progressed during the treatment. In the CCRT group, $6(60 \%)$ of 10 patients developed local relapse (3 in 3D-CRT group), 2 patients experienced a relapse in the nasal cavity within his previous radiation field, and 1 patient experienced disease 


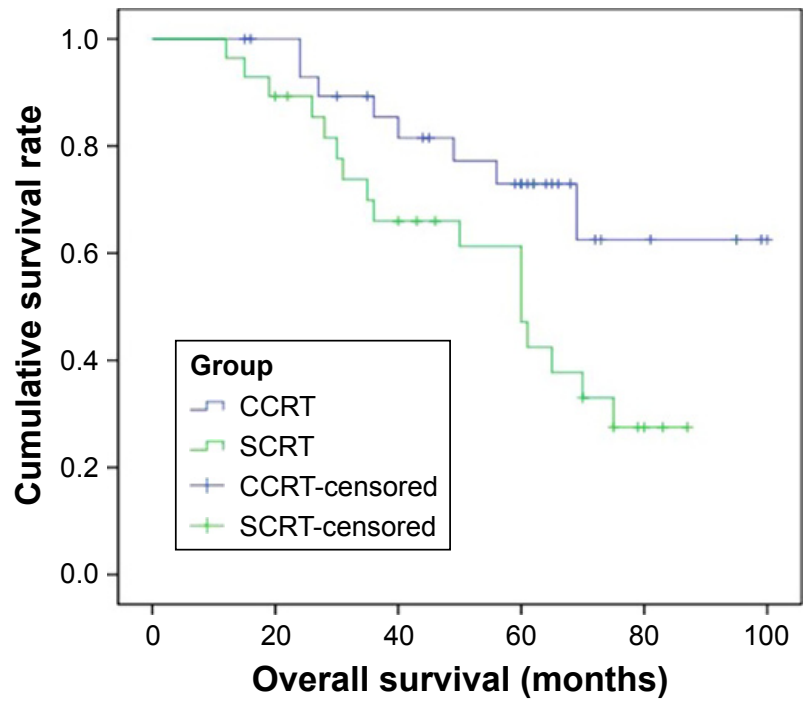

Figure I Comparison of overall survival between the CCRT and SCRT groups $(P=0.029)$.

Abbreviations: CCRT, concurrent chemoradiotherapy; SCRT, sequential chemotherapy followed by radiotherapy.

progression in the laryngopharyngeal area just outside the border of the radiation field. Distant relapses were observed in another $4(40 \%)$ patients; 1 in the colon, 2 in the breast, and 1 in the liver. Among them, 1 patient was in the IMRT group and 3 patients were in the 3D-CRT group. In the SCRT group, $12(80 \%)$ of 15 patients developed local relapse (5 in the IMRT group and 7 in the 3D-CRT group). Six patients experienced disease progression in the nasal cavity, 3 patients in the nasopharynx, and 3 patients in the left maxillary sinus

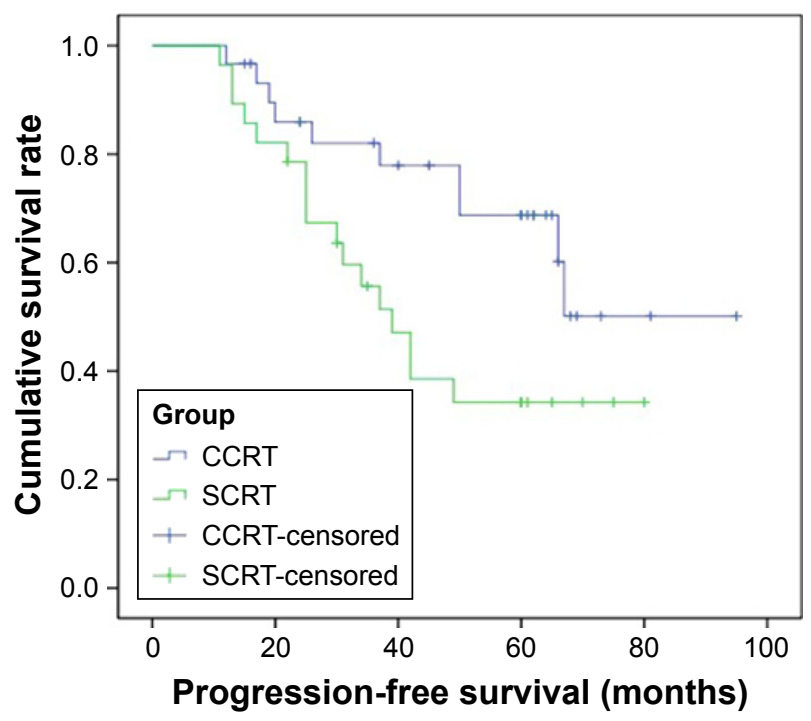

Figure 2 Comparison of progression-free survival between the CCRT and SCRT groups $(P=0.030)$.

Abbreviations: CCRT, concurrent chemoradiotherapy; SCRT, sequential chemotherapy followed by radiotherapy.
Table 2 Patterns of failure for CCRT and SCRT group patients

\begin{tabular}{lll}
\hline Patterns of failure & CCRT, $\mathbf{n}$ & SCRT, $\mathbf{n}$ \\
\hline Local relapse & 6 & 12 \\
In the nasal cavity & 2 & 6 \\
In laryngopharyngeal area & $\mathrm{I}$ & 0 \\
In nasopharyngeal & 2 & 3 \\
In the left maxillary sinus & $\mathrm{I}$ & 3 \\
Distant relapse & 4 & 3 \\
Liver & $\mathrm{I}$ & $\mathrm{I}$ \\
Breast & 2 & 0 \\
Lung & 0 & $\mathrm{I}$ \\
Skin & 0 & $\mathrm{I}$ \\
Colon & $\mathrm{I}$ & 0 \\
\hline
\end{tabular}

Abbreviations: CCRT, concurrent chemoradiotherapy; SCRT, sequential chemotherapy followed by radiotherapy.

within his prior radiation field. There was distant relapse in $2(13.3 \%)$ patients; 1 in the lung and 1 in the liver. Combined local and distant relapse was found in $1(6.7 \%)$ patient. She experienced relapse in the nasal cavity and had multiple skin lesions (Table 2). Local failure was more frequent in the SCRT group (12 of 15 [80\%] with recurrence) than in the CCRT group (6 of 10 [60\%] with recurrence). However, there was no significant difference between the 2 groups $(P=0.236)$. The 5-year LRFS rate of the CCRT and SCRT was $78.9 \%$ and $45.7 \%$, respectively, which was significant $(P=0.026)$ (Figure 3).

\section{Toxicity}

Treatment-related toxicities are listed in Table 3. There was no case of grade III or IV anemia or thrombocytopenia in

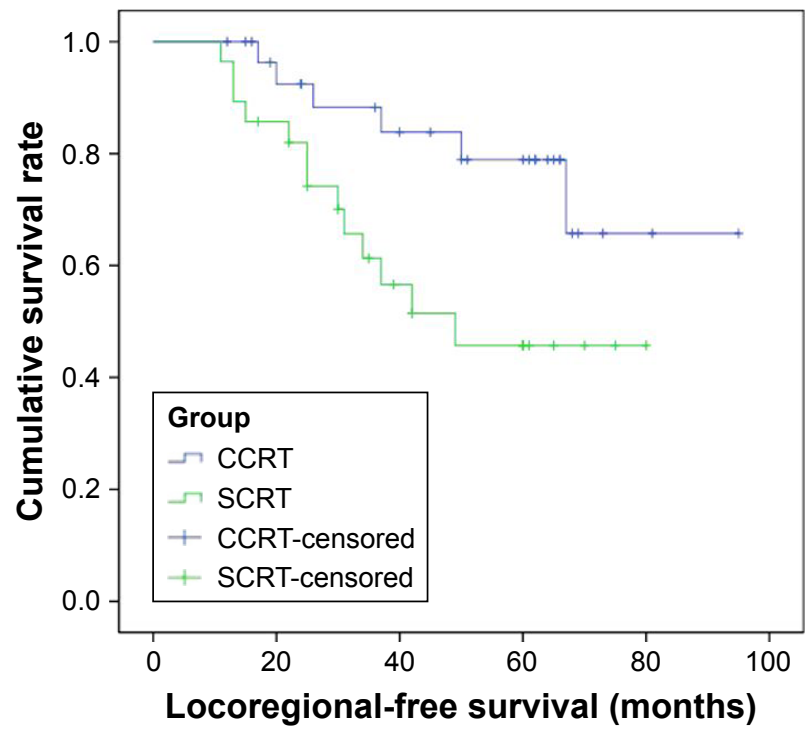

Figure 3 Comparison of locoregional-free survival between the CCRT and SCRT groups $(P=0.026)$.

Abbreviations: CCRT, concurrent chemoradiotherapy; SCRT, sequential chemotherapy followed by radiotherapy. 
Table 3 Adverse event grade by treatment group

\begin{tabular}{|c|c|c|c|c|c|c|c|c|c|c|}
\hline \multirow[t]{2}{*}{ Toxicity } & \multicolumn{4}{|c|}{ CCRT } & \multicolumn{4}{|c|}{ SCRT } & \multirow[t]{2}{*}{$\chi^{2}$ value } & \multirow[t]{2}{*}{$P$-value } \\
\hline & $\mathrm{I}$ & 2 & 3 & 4 & $\mathbf{I}$ & 2 & 3 & 4 & & \\
\hline \multicolumn{11}{|l|}{ Hematologic } \\
\hline Anemia & 3 & 5 & 0 & 0 & 4 & 0 & 0 & 0 & 4.286 & 0.081 \\
\hline Leukopenia & II & 8 & 6 & 5 & 12 & 10 & 5 & 0 & 5.213 & 0.157 \\
\hline Thrombocytopenia & 6 & 4 & 0 & 0 & 5 & 1 & 0 & 0 & 0.950 & 0.330 \\
\hline \multicolumn{11}{|l|}{ Nonhematologic } \\
\hline Nausea & 9 & 12 & 6 & 0 & 7 & 9 & 3 & 0 & 0.296 & 0.862 \\
\hline Vomiting & 12 & 9 & 6 & 3 & 17 & 7 & 4 & 0 & 4.448 & 0.217 \\
\hline Mucositis & 8 & 13 & 7 & 2 & 15 & 10 & 3 & 0 & 6.060 & 0.109 \\
\hline
\end{tabular}

Abbreviations: CCRT, concurrent chemoradiotherapy; SCRT, sequential chemotherapy followed by radiotherapy.

the 2 groups. Mucositis was commonly associated with RT in both the SCRT and CCRT groups. However, most cases were grades I and II. Grade III mucositis rates were $23.3 \%$ and $10.7 \%$, respectively. In $5(16.7 \%)$ CCRT patients, there was grade IV leukopenia. Even so, they continue treatment without interruption. A total of 11 patients $(18.9 \%$; 6 of 30 [20\%] in the CCRT group and 5 of 28 [17.8\%] in the SCRT group) experienced grade III leukopenia. Overall, there were no significant differences in the toxicities between the 2 groups during treatment. All patients with this condition fully recovered with supportive care. Moreover, all patients completed the planned course of treatment without dose reduction, and there was no treatment-related mortality in either the CCRT or the SCRT group. No patient suffered from the following problems: pancreatitis, elevated serum glucose level, or blood clotting disorder.

\section{Discussion}

Considering the poor outcome of ENKTL treated with RT and chemotherapy alone, it is reasonable to combine the 2 therapies to improve treatment efficacy. CCRT and SCRT are both widely used in local ENKTL treatment. However, there is still no consensus concerning whether CCRT or SCRT is preferred.

Theoretically, RT and chemotherapy performed concurrently can interact with each other and achieve a better outcome. Chemotherapy can increase the sensitivity of tumor cells to RT, and RT can in turn enhance the cytotoxicity of chemotherapy, which can exert a more potent effect on local tumors, avoiding tumor cell accelerated proliferation. Moreover, chemotherapy can kill tiny, hidden metastatic lesions that already exist, thereby reducing the distant metastatic rate and increasing the RT treatment outcome effectiveness. Two prospective trials have demonstrated a beneficial role for CCRT in the treatment of stage I/II nasal
ENKTL. The estimated 3-year PFS and OS for CCRT were, respectively, as high as $85.19 \%$ and $86.28 \%$ in a Korean Phase II trial. ${ }^{12}$ Moreover, CCRT also yielded high 5 -year PFS (67\%) and OS (73\%) in the JCOG0211 study. ${ }^{13}$ So CCRT is recommended as the first-line treatment for this early-stage disease.

However, 2 recent clinical studies in Korea showed negative results that there were no survival differences between the CCRT and SCRT. Lee et a ${ }^{14}$ retrospectively analyzed the outcomes of 46 patients with stage I/II nasal ENKTL, where 33 patients were treated with SCRT (50.4 Gy in 28 fractions) and 13 patients were treated with CCRT (40 Gy in 20 fractions). There was no statistically significant difference in survival outcome between the SCRT and CCRT groups. Lee et $\mathrm{al}^{15}$ summarized their clinical experience of patients with stage I/II nasal ENKTL, treated using SCRT or CCRT. Forty-three patients with stage I/II nasal ENKTL, who received SCRT (16 patients) or CCRT (27 patients), were included in the analysis. There were no statistically significant differences between the SCRT and CCRT groups with regard to the 3 -year PFS (56\% vs $41 \%, P=0.823$ ) and 3 -year OS ( $75 \%$ vs $59 \%, P=0.670)$.

In our study, we compared the curative effect of SCRT and CCRT of nasal ENKTL in our center. In contrast with the earlier results, our findings showed that the 5-year OS (72.9\% vs $47.1 \%, P=0.029)$ and PFS $(68.8 \%$ vs $34.2 \%$, $P=0.030$ ) rates were significantly better in CCRT group than those in SCRT group. The 5-year LRFS of the CCRT and SCRT was $78.9 \%$ and $45.7 \%$, respectively, and there was a significant difference between the 2 groups $(P=0.026)$. Yang et $\mathrm{al}^{16}$ compared CCRT with SCRT in a group of patients with early-stage nasal ENKTL. There were statistically significant differences between the CCRT and SCRT groups with regard to the 5 -year OS ( $80.8 \%$ vs $54.3 \%, P=0.05)$ and 5 -year PFS ( $75.8 \%$ vs $43.3 \%, P=0.05$ ). Our results are similar to those reported by Yang. The improved PFS and OS in the CCRT group might be partly explained by the better potential minimal distant disease eradication of CCRT, which needs to be validated and explored further.

Several studies have reported a beneficial role for early or upfront RT in good local control. ${ }^{13,17,18}$ The local control rate of our study in the CCRT group was lower than that in the SCRT group (30\% vs $58.8 \%$, respectively), which demonstrates that upfront RT in the CCRT group is crucial in achieving a good local control. Kim et a ${ }^{12}$ initiated a multicenter Phase II trial that included 30 patients with untreated localized nasal NK/T-cell lymphoma, who were treated with RT (40 Gy) administered concurrently with weekly cisplatin, 
followed by 3 cycles of etoposide, ifosfamide, cisplatin, dexamethasone chemotherapy. The estimated 3-year local control rate was $93 \%$. Lee et $\mathrm{al}^{14}$ previously reported that the CCRT group tended to show a better local recurrence-free survival and distant metastasis-free survival than the SCRT group. The survival analysis indicated that the rate of 5-year LRFS in the CCRT group was significantly higher than that in the SCRT group ( $94.1 \%$ vs $56.7 \%, P=0.01$ ). Our study showed that the 5-year LRFS rate of the CCRT (78.9\%) is superior to the SCRT (45.7\%), and there was significant difference between the 2 groups $(P=0.026)$.

Although the patients of the CCRT group had more serious leukopenia and gastrointestinal reaction, all patients with this complication fully recovered with supportive care. All patients completed the planned course of treatment without interruption, and there was no treatment-related mortality.

We think some factors in our study may be favorable and have affected the treatment outcomes. First, more patients in the CCRT group received higher RT doses than those in the SCRT group in our study. Until recently, an RT dose of $<50$ Gy was recommended for localized ENKTL despite the lack of high-level evidence. You et al ${ }^{17}$ administered higher RT doses (54-60 Gy) and achieved an 83.3\% failure-free survival rate at 5 years. Second, there were more patients who received large involved-field RT in the CCRT group than in the SCRT group in our study. Isobe et $\mathrm{al}^{9}$ found that patients who received large involved-field RT to all macroscopic lesions and sites of potential contiguous spread achieved better local control than patients who received low-dose or involved-field RT. Additionally, the relationship between the radiation dose and field and locoregional control or survival has been confirmed by several other studies. ${ }^{18,19}$

Third, there were 20 IMRT cases $(66.7 \%)$ in the CCRT group and only $9(32.1 \%)$ in the SCRT group. Because IMRT demonstrated the advantage of better dose coverage, ${ }^{20}$ the relatively high proportion of IMRT in the CCRT group may have improved the survival outcome. On the contrary, there were still several limitations to the present study including the retrospective nature of the study design and the small sample size.

\section{Conclusion}

We demonstrated higher 5-year OS, PFS, and LRFS in the CCRT group. CCRT may be an effective and tolerated treatment in the treatment of stage I/II nasal ENKTL. These results require further investigation in larger trials.

\section{Disclosure}

The authors report no conflicts of interest in this work.

\section{References}

1. Oshimi K. Progress in understanding and managing natural killer-cell malignancies. Br J Haematol. 2007;139(4):532-544.

2. Suzuki R, Takeuchi K, Ohshima K, Nakamura S. Extranodal NK/T-cell lymphoma: diagnosis and treatment cues. Hematol Oncol. 2008; 26(2):66-72.

3. Sun J, Yang Q, Lu Z, et al. Distribution of lymphoid neoplasms in China: analysis of 4,638 cases according to the World Health Organization classification. Am J Clin Pathol. 2012;138(3):429-434.

4. Jain S, Zain J, O'Connor O. Novel therapeutic agents for cutaneous T-Cell lymphoma. J Hematol Oncol. 2012;17(5):24.

5. Lim ST, Hee SW, Quek R, et al. Comparative analysis of extra-nodal NK/T-cell lymphoma and peripheral T-cell lymphoma: significant differences in clinical characteristics and prognosis. Eur J Haematol. 2008;80(1):55-60.

6. Lin JC, Wang WY, Chen KY, et al. Quantification of plasma EpsteinBarr virus DNA in patients with advanced nasopharyngeal carcinoma. N Engl J Med. 2004;350(24):2461-2470.

7. Hohaus S, Santangelo R, Giachella M, et al. The viral load of EpsteinBarr virus (EBV) DNA in peripheral blood predicts for biological and clinical characteristics in Hodgkin lymphoma. Clin Cancer Res. 2011; 17(9):2885-2892.

8. Li YX, Yao B, Jin J, et al. Radiotherapy as primary treatment for stage IE and IIE nasal natural killer/T-cell lymphoma. J Clin Oncol. 2006;249(1):181-189.

9. Isobe K, Uno T, Tamaru J, et al. Extranodal natural killer/T-cell lymphoma, nasal type: the significance of radiotherapeutic parameters. Cancer. 2006;106(3):609-615.

10. Ribrag V, Ell Hajj M, Janot F, et al. Early locoregional high-dose radiotherapy is associated with long-term disease control in localized primary angiocentric lymphoma of the nose and nasopharynx. Leukemia. 2001;15(7):1123-1126.

11. Sobrevilla-Calvo P, Meneses A, Alfaro P, Bares JP, Amador J, Reynoso EE. Radiotherapy compared to chemotherapy as initial treatment of angiocentric centrofacial lymphoma (polymorphic reticulosis). Acta Oncol. 1993;32(1):69-72.

12. Kim SJ, Kim K, Kim BS, et al. Phase II trial of concurrent radiation and weekly cisplatin followed by VIPD chemotherapy in newly diagnosed stage IE to IIE, nasal, extranodal NK/T-cell lymphoma: consortium for improving survival of lymphoma study. J Clin Oncol. 2009;27(35):6027-6032.

13. Yamaguchi M, Tobinai K, Oguchi M, et al. Concurrent chemoradiotherapy for localized nasal natural killer/T-cell lymphoma: an updated analysis of the Japan clinical oncology group study JCOG0211. J Clin Oncol. 2012;30(32):4044-4046.

14. Lee HJ, Lee SW, Suh C, et al. Treatment outcome of nasal natural killer/T-cell lymphoma. Radiat Oncol J. 2011;29(3):174-180.

15. Lee J, Kim CY, Park YJ, Lee NK. Sequential chemotherapy followed by radiotherapy versus concurrent chemoradiotherapy in patients with stage I/II extranodal natural killer/T-cell lymphoma, nasal type. Blood Res. 2013;48(4):274-281.

16. Yang Y, Zhang YJ, Sun Y, et al. Preliminary outcome of concurrent chemoradiation therapy in patients with early stage extranodal nasal type NK/T-cell lymphoma. Chin J Cancer Prev Treat. 2012;19(6): 378-381.

17. You JY, Chi KH, Yang MH, et al. Radiation therapy versus chemotherapy as initial treatment for localized nasal natural killer (NK)/T-cell lymphoma: a single institute survey in Taiwan. Ann Oncol. 2004;15(4): 618-625.

18. Huang MJ, Jiang Y, Liu WP, et al. Early or up-front radiotherapy improved survival of localized extranodal NK/T-cell lymphoma, nasaltype in the upper aerodigestive tract. Int J Radiat Oncol Biol Phys. 2008;70(1):166-174 
19. Cox JD, Stetz J, Pajak TF. Toxicity of the radiation therapy oncology group (RTOG) and the European organization for research and treatment of cancer (EORTC). Int J Radiat Oncol Biol Phys. 1995;31(5): 1341-1346.
20. Shen $\mathrm{Q}, \mathrm{Ma} \mathrm{X}, \mathrm{Hu} \mathrm{W}$, et al. Intensity-modulated radiotherapy versus three-dimensional conformal radiotherapy for stage I/II natural killer T-cell lymphoma nasal type: dosimetric and clinical results. Radiat Oncol. 2013;8:152.

\section{Publish your work in this journal}

OncoTargets and Therapy is an international, peer-reviewed, open access journal focusing on the pathological basis of all cancers, potential targets for therapy and treatment protocols employed to improve the management of cancer patients. The journal also focuses on the impact of management programs and new therapeutic agents and protocols on

\section{Dovepress}

patient perspectives such as quality of life, adherence and satisfaction. The manuscript management system is completely online and includes a very quick and fair peer-review system, which is all easy to use. Visit http://www.dovepress.com/testimonials.php to read real quotes from published authors.

Submit your manuscript here: http://www.dovepress.com/oncotargets-and-therapy-journal 